hep-th/0107124

July 2001

\title{
Cosmological Evolution in a Type-0 String Theory
}

\author{
G. A. Diamandis and B. C. Georgalas \\ Physics Department, Nuclear and Particle Physics Section, University of Athens, \\ Panepistimioupolis GR 157 71, Ilisia, Athens, Greece. \\ N. E. Mavromatos \\ Department of Physics, Theoretical Physics, King's College London, \\ Strand, London WC2R 2LS, United Kingdom. \\ E. Papantonopoulos and I. Pappa \\ Department of Physics, National Technical University of Athens, \\ Zografou Campus GR 157 80, Athens, Greece.
}

\begin{abstract}
We study the cosmological evolution of a type-0 string theory by employing noncriticality, which may be induced by fluctuations of the D3 brane worlds. We check the consistency of the approach to $\mathcal{O}\left(\alpha^{\prime}\right)$ in the corresponding $\sigma$-model. The ten-dimensional theory is reduced to an effective four-dimensional model, with only time dependent fields. We show that the four-dimensional universe has an inflationary phase and graceful exit from it, while the other extra dimensions are stabilized to a constant value, with the fifth dimension much larger than the others. We pay particular attention to demonstrating the rôle of tachyonic matter in inducing these features. The Universe asymptotes, for large times, to a non-accelerating linearly-expanding Universe with a time-dependent dilaton and a relaxing to zero vacuum energy a lá quintessence.
\end{abstract}




\section{Introduction}

Interest in type-0 string theories has recently arisen due to their connection to fourdimensional $S U(N)$ gauge theory via appropriate dualities [1]. The type-0 string model contains electrically-charged three branes, $N$ of which may be stuck on top of each other to construct the dual of a gauge theory. An interesting feature of these non-supersymmetric theories is the appearance of tachyonic matter excitations. These, however, do not lead to an instability, since they couple to the appropriate five-form field strength that drives the tachyon to a non-trivial vacuum expectation value capable of reversing the original (negative) sign of the mass-squared.

Irrespective of the connection with dual gauge theories, the presence of tachyonic matter in type-0 string theories has important consequences in implying a cosmological evolution of the brane of inflationary type [2]. The rôle of tachyonic matter in inducing non-trivial cosmological evolution in two-dimensional string models has been studied previously [3], with interesting conclusions on the possibility of inflationary phase as well as 'graceful' exit from it [4]. It should be stressed, however, that the graceful exit issue has been resolved only upon invoking non-criticality of the underlying string theory, which is arguably consistent with the non-equilibrium nature of the inflationary phase. An important issue in such noncritical string analyses is the identification of target time with the Liouville mode [5], the consistency of which with the presence of an inflationary phase and the eventual graceful exit is a highly non-trivial issue.

Such a consistency is equivalent to demonstrating the existence of a solution of a system of equations which represent a generalization of the conformal invariance conditions in the case of a non-critical Liouville string, as a result of the restoration of conformal invariance after Liouville dressing [6]. An interesting physical issue is what causes the non-criticality of the type-0 string. At present, the best scenario we can offer are the quantum fluctuations of the three brane solitonic structures $\llbracket 7,8$. In General Relativity there are no Rigid Objects, 
due to general covariance, and so recoil fluctuations of the brane worlds are expected in general. Moreover reconciliation with quantum mechanics requires the incorporation of position and momentum uncertainties of the brane worlds.

Under the identification of the Liouville mode with the target time, the induced noncriticality leads to interesting predictions on the possibility of an inflationary phase of the fluctuating brane [9]. However, the interesting issue is the exit from such a phase. It appears that, apart from the graviton and dilaton fields on the brane, the issue of graceful exit from the inflationary phase requires the presence of additional matter excitations. It is the purpose of this work to analyse an effective theory on a three-brane in the context of a non-critical type-0 string theory, with the aim of demonstrating such a graceful exit from the inflationary phase. As mentioned above, the non-criticality will be associated with quantum fluctuations of the embedded three-branes.

We consider a non-critical ten-dimensional string theory of type-0, and we dimensionally reduce the ten-dimensional $\mathcal{O}\left(\alpha^{\prime}\right)$ string-effective action to a four-dimensional space-time on a 3-brane world, assuming that all fields depend only upon time. We choose two different fields to parametrize the internal space. The first field sets the scale of the fifth dimension, while the other parametrize a flat five dimensional space. In the same way, we dimensionally reduce the modified (because of the off-criticality) $\beta$-functions of the ten-dimensional theory, to the effective four-dimensional $\beta$-functions, assuming a Robertson-Walker form of the four-dimensional metric. Liouville dressing of the non-critical theory should restore conformal invariance of the $\sigma$-model, which results in a set of generalized conformal invariance conditions. Upon the identification of the Liouville mode with the target time, which eliminates scenaria with two target time-like coordinates, such conditions become equivalent to 'equations of motion' for the appropriate fields.

These equations, supplemented by the Curci-Paffuti equation, stemming from worldsheet renormalizability of the $\sigma$-model, are studied in detail. At present, they appear to 
be too complicated to be solved analytically. We thus follow a systematic method, that of quasi-linear systems, to solve these equations numerically.

The main results of such an analysis may then be summarized as follows: our model clearly exhibits an inflationary phase and, most importantly, a graceful exit from it. The supercriticality, as well as the tachyonic matter, play a crucial rôle to this effect. Moreover, there is stabilization of the internal space. The fifth dimension contracts during inflation and then freezes to a constant value, while the other five internal dimensions also freeze to a constant value, which in the solution we present in section four, is different from the first, indicating the possibility of obtaining different scales for the extra dimensions.

The structure of the article is as follows: in section two we give a brief review of the Liouville formalism under the identification of the Liouville mode with the target time. In section three we discuss the type-0 string theory and we derive the equations of motion of the effective non-critical four-dimensional theory. In section four, we describe the method

for solving numerically the system of the differential equations describing the equations of motion and we support our findings by analytic arguments on the asymptotic behaviour of the solutions. We present the numerical results and we discuss their physical significance. Finally, in section five, we present our conclusions and outlook. Some important formal properties of the dilaton field in the context of Liouville strings are discussed in an appendix.

\section{Liouville String Formalism}

We commence our analysis with a brief review of the Liouville dressing procedure for non-critical strings, with the Liouville mode viewed as a local world-sheet renormalization group scale [5]. Consider a conformal $\sigma$-model, described by an action $S^{*}$ on the worldsheet $\Sigma$, which is deformed by (non conformal) deformations $\int_{\Sigma} g^{i} V_{i} d^{2} \sigma$, with $V_{i}$ appropriate 
vertex operators.

$$
S_{g}=S^{*}+\int_{\Sigma} g^{i} V_{i} d^{2} \sigma
$$

The non-conformal nature of the couplings $g^{i}$ implies that their (flat)world sheet renormalization group $\beta$-functions, $\beta^{i}$, are non-vanishing. The generic structure of such $\beta$ functions, close to a fixed point, $\left\{g^{i}=0\right\}$ reads:

$$
\beta^{i}=\left(h_{i}-2\right) g^{i}+c_{j k}^{i} g^{j} g^{k}+o\left(g^{3}\right)
$$

In the context of Liouville strings, world-sheet gravitational dressing is required. The "gravitationally"-dressed couplings, $\lambda^{i}(g, t)$, which from our point of view correspond to renormalized couplings in a curved space, read to $O\left(g^{2}\right)$ [6, 10]:

$$
\lambda^{i}(g, t)=g^{i} e^{\alpha_{i} t}+\frac{\pi}{Q \pm 2 \alpha_{i}} c_{j k}^{i} g^{j} g^{k} t e^{\alpha_{i} t}+O\left(g^{3}\right), \quad Q^{2}=\frac{1}{3}\left(c-c^{*}\right)
$$

where $t$ is the (zero mode) of the Liouville mode, $c^{*}$ is a critical (fixed point) value of the central charge, about which we perturb the theory, $Q^{2}$ is the central charge deficit, and $\alpha_{i}$ are the gravitational anomalous dimensions:

$$
\alpha_{i}\left(\alpha_{i}+Q\right)=h_{i}-2 \quad \text { for } \quad c \geq c^{*}
$$

Below we shall concentrate exclusively to the supercritical string case, $Q^{2} \geq 0$, which from the point of view of identifying the Liouville mode with target time, corresponds to a Minkowskian signature spacetime manifold. The fixed point value $c^{*}$ is normalized such that for superstrings we are dealing with here, one has $Q^{2}=\frac{1}{2}(d-9)$, in the special case of superstrings with non critical space time dimensionality $(d)$.

Due to the renormalization (2.3), the critical-string conformal invariance conditions, amounting to the vanishing of flat-space $\beta$-functions, are now substituted by:

$$
\ddot{\lambda}^{i}+Q \dot{\lambda}^{i}=-\beta^{i}(\lambda) \quad \text { for } c \geq c^{*}
$$

where the notation $\beta^{i}(\lambda)$ denotes flat-world-sheet $\beta$-functions but with the formal substitution $g^{i} \rightarrow \lambda^{i}(g, t)$. Note the minus sign in front of the flat world sheet $\beta$-functions $\beta^{i}$ in 
(2.5), which is characteristic of the supercriticality of the string [6, 10]. As we see later, the sign will be crucial for the existence of acceptable inflationary solutions demonstrating graceful exit from the exponential expansion phase. Upon the identification of the Liouville mode with the target time the dot denotes temporal derivative.

A highly non-trivial feature of the $\beta^{i}$ functions is the fact that they are expressed as gradient flows in theory space [11, [12], i.e. there exists a 'flow' function $\mathcal{F}[g]$ such that

$$
\beta^{i}=\mathcal{G}^{i j} \frac{\delta F[g]}{\delta g^{j}}
$$

where $\mathcal{G}^{i j}$ is the inverse of the Zamolodchikov metric in theory space [11], which is given by appropriate two-point correlation functions between vertex operators $V^{i}$. In the case of stringy $\sigma$-models the flow function $\mathcal{F}$ may be identified [12 with the running central charge deficit $Q^{2}=c[g]-c^{*}$, where $c[g]$ is the central charge of the deformed theory, and $c^{*}$ one of its critical values (conformal point) about which the theory is perturbed by means of the operators $V^{i}$.

It is then interesting to notice that, upon the identification of the Liouville mode with the target time [5], the form of equations (2.5), upon the condition (2.6), is reminiscent of inflaton equations in standard Cosmology, with the rôle of the inflaton potential being played by the flow function $Q^{2}[g]$ in theory space, and that of the Hubble parameter $H$ by its square root $Q[g]$. There is an important difference, though, as compared with the inflaton case, in the sense that here all the field modes seem to exhibit this behaviour.

An important comment we would like to make concerns the possibility of deriving the set of equations (2.5) from a target space action. This issue has been discussed in the affirmative in [13, where it was shown that the set of equations (2.5) satisfies the Helmholtz conditions for the existence of an action in the 'space of couplings' $\left\{g^{i}\right\}$ of the non-critical string. The property (2.6) is crucial to this effect. Upon the identification of target time with the Liouville mode [5] this action becomes identical with the target space action describing the off-shell dynamics of the Liouville string. We should stress the 
fact that the action is off shell, in the sense that the on-shell conditions correspond to the vanishing of the $\beta$-functions $\beta^{i}$. In our case $\beta^{i} \neq 0$, and the identification of the Liouville mode with the target time implies that the space time graviton $\beta$-function on the right hand side of (2.5), as well as other target-space tensorial structures, appearing inside the $\beta^{i}$ functions for the various modes, contain temporal (Liouville) components as well.

In this respect, our non-equilibrium Liouville string approach to the temporal evolution should be contrasted with the interpretation of a Liouville string as a critical equilibrium string living in a space time with one extra dimension. In that case the corresponding $\beta$-functions of the Liouville-dressed theory would satisfy classical equations of motion. As mentioned above, in our approach the conditions describing the restoration of conformal invariance by means of Liouville dressing are not to be interpreted as classical equations

of motion of a string living in a space-time with one extra target dimension. Thus our analysis below should be distinguished from previous analysis on Liouville cosmology [14].

A generic feature of Liouville dynamics, is that in terms of the world-sheet action, the normalization of the Liouville kinetic term can always be arranged (by choosing appropriate counterterms) to correspond to a target spacetime of Friedman-Robertson-Walker (FRW) type; i.e. the time-like metric component (under the assumption that the Liouville mode is time) is:

$$
G_{00}=-1
$$

We remind the reader that the Minkowskian signature is due to supercriticality $\left(3 Q^{2}=\right.$ $\left.c-c^{*} \geq 0\right)$ assumption. This will be understood in what follows.

\section{Non-Criticality Induced in Type-0 String Theory}

We will start our discussion with the ten-dimensional type-0 string theory. The effective ten-dimensional target space action of the type-0 String has the form, to $\mathcal{O}\left(\alpha^{\prime}\right)$ in the Regge 
slope $\alpha^{\prime}[1]$ :

$$
\begin{aligned}
S= & \int d^{10} x \sqrt{-G}\left[e ^ { - 2 \Phi } \left(R+4\left(\partial_{M} \Phi\right)^{2}-\frac{1}{4}\left(\partial_{M} T\right)^{2}-\frac{1}{4} m^{2} T^{2}\right.\right. \\
& \left.\left.-\frac{1}{12} H_{M N P}^{2}\right)-\frac{1}{4}\left(1+T+\frac{T^{2}}{2}\right)\left|\mathcal{F}_{M N P \Sigma T}\right|^{2}\right]
\end{aligned}
$$

where capital Greek letters denote ten-dimensional indices, $\Phi$ is the dilaton, $H_{M N P}$ denotes the field strength of the antisymmetric tensor field, which we shall ignore in the present work, $T$ is the tachyon field of mass $m^{2}<0$. In our analysis we have ignored higher-order terms in the tachyon potential. The quantity $\mathcal{F}_{M N P \Sigma T}$ denotes the appropriate five-form of type- 0 string theory, which couples to the tachyon field in the Ramond-Ramond (RR) sector via the function $f(T)=1+T+\frac{1}{2} T^{2}$.

From (3.1) one sees easily the important rôle of the five-form $\mathcal{F}$ in stabilizing the ground state. Due to its special coupling with the quadratic $T^{2}$ term in RR sector of the theory, it yields an effective mass term for the tachyon which is positive, despite the originally negative $m^{2}$ contribution [1].

To go off criticality we add the following term to the action (3.1)

$$
-\int d^{10} x \sqrt{-G} e^{-2 \Phi} Q^{2}
$$

The sign of $Q^{2}$ is positive if one assumes supercriticality of the string, which we shall do here [15, 6, 1]. There may be various reasons for having such a term in the effective action. One concrete example of induced super-criticality in brane-world scenaria is the presence of impulse action (recoil) on the D3-world brane as a result of either quantum fluctuations or scattering with other heavy defects or (a macroscopic number of) closed string states [7]. As argued in those works the impulse deformations of the pertinent $\sigma$-model are relevant from a world-sheet renormalization-group view point, and as such require Liouville dressing [6]. The Liouville mode may be identified with the target time in a way consistent with the restoration of conformal invariance. In general, there may be other microscopic reasons for the induction of non-criticality in string theory, all related 
to some sort of non-equilibrium processes, which are expected to play a dominant rôle in early Universe Cosmologies. For the purposes of the present article, therefore, we shall not specify further the precise microscopic origin of such non-criticality, apart from associating it generically with fluctuations of the brane worlds. In this sense we may assume $Q$ to be a rather 'phenomenological' parameter, to be determined by consistency with the conformal invariance conditions of the Liouville-dressed [6] $\sigma$-model. For our purposes we shall restrict ourselves to $\mathcal{O}\left(\alpha^{\prime}\right)$ string effective actions.

In general, $Q^{2}$ depends on the $\sigma$-model backgrounds fields, being the analogue of Zamolodchikov's $C$-function [11]. Assuming renormalizability, implies that any explicit time-dependence of $Q$ will be absorbed in renormalized couplings $g^{I}$ :

$$
\frac{d}{d t} Q=\frac{\partial}{\partial t} Q+\beta^{I} \partial_{I} Q=0
$$

However, the true set of non-marginal couplings in string theory is actually infinite. Moreover, in our case, there are non conformal contributions from world-sheet boundaries as well, expressing, for instance, recoil of the (quantum) fluctuating D3 brane world.

Formally, such fluctuations may be approximated semi-classically by impulse-type logarithmic conformal deformations at a $\sigma$-model level [7], which have recently been shown to exist also in theories with world-sheet fermions [8], such as the type-0 string theories under consideration [1]. Such contributions can only be computed approximately at present, for weakly coupled strings [7, 8, 16], and thus are not taken into account explicitly in the effective 'phenomenological' approach adopted here, where only the bulk (closed-string sector) contributions will be considered in a detailed manner. We denote such contributions by an upper case small Latin index $\left\{g^{i}\right\}$, in contrast with the upper case capital index $g^{I}$ which denotes the complete set of non-conformal deformations.

In view of (3.3) this implies that in our case we should consider $Q\left(t, g^{i}\right)$ as depending explicitly on time (renormalization-group scale $t$ ), in addition to its implicit dependence through the renormalized couplings $g^{i}$. This explicit time dependence simply expresses the 
contributions to $Q$ from the rest of the non-conformal modes. Its form, then, as a function of time $t$, should be determined by demanding consistency with the generalized conformalinvariance conditions (2.5) obtained for the problem at hand from the action (3.1). This is the approach we shall assume from now on.

To this end, we need first to consider the dimensional reduction of the ten-dimensional action to the four-dimensional space-time on the brane. This procedure is achieved by assuming the following ansatz for the ten-dimensional metric:

$$
G_{M N}=\left(\begin{array}{ccc}
g_{\mu \nu}^{(4)} & 0 & 0 \\
0 & e^{2 \sigma_{1}} & 0 \\
0 & 0 & e^{2 \sigma_{2}} I_{5 \times 5}
\end{array}\right)
$$

where lower-case Greek indices are four-dimensional space time indices, and $I_{5 \times 5}$ denotes the $5 \times 5$ unit matrix. We have chosen two different scales for internal space. The field $\sigma_{1}$ sets the scale of the fifth dimension, while $\sigma_{2}$ parametrize a flat five dimensional space. In the context of homogeneous cosmological models, we are dealing with here, the fields $g_{\mu \nu}^{(4)}$ are of Robertson-Walker type, and $\sigma_{i}, i=1,2$ are assumed to depend on the time $t$ only.

By varying the effective action with respect to the five-form one obtains the corresponding $\beta$-function:

$$
\tilde{\beta}_{5}=\nabla_{M}\left[\left(1+T+\frac{T^{2}}{2}\right) \mathcal{F}^{M N P \Sigma T}\right]
$$

It is important to notice that in our approach we consider as a source of non-criticality for the underlying string theory only quantum fluctuations of the D3 brane resulting in the non-vanishing of the renormalization group $\beta$-functions of the string multiplet, i.e. graviton, dilaton and tachyon fields. The deformation corresponding to the five-form [1] is assumed conformal. This is associated with that fact that this field is related to the RR electric flux of the D-branes, and as such is not affected by the (recoil) quantum fluctuations. Moreover, for our purposes in this work, which is to demonstrate an inflationary phase and graceful exit from it, the essential rôle is played by the string multiplet fields, with the five-form 
flux field serving the sole purpose of tachyon stabilization. This implies the vanishing of the left-hand side of (3.5):

$$
0=\nabla_{M}\left[\left(1+T+\frac{T^{2}}{2}\right) \mathcal{F}^{M N P \Sigma T}\right]
$$

Moreover, for simplicity we shall assume a configuration for which the five-form acquires non-vanishing values only along one extra dimension:

$$
\mathcal{F}_{M N P \Sigma T}=\varepsilon_{0 \alpha \beta \gamma \delta} f_{5}(t)
$$

where the lower-case Greek letters denote four-dimensional indices.

The ten-dimensional Weyl-anomaly coefficients (generalized $\beta$-functions [17]) for the $\sigma$-model corresponding to propagation in a type-0 string background read, to $\mathcal{O}\left(\alpha^{\prime}\right)$ :

$$
\begin{aligned}
\tilde{\beta}_{M N}^{G}= & \left\{R_{M N}+2 \Phi_{; M N}-\frac{1}{4} T_{; M} T_{; N}\right\}+ \\
& \frac{1}{2} e^{2 \Phi} f(T)\left[G_{M N}\left(\mathcal{F}_{K \Lambda P \Sigma T}\right)^{2}-10 \mathcal{F}_{M K \Lambda P \Sigma} \mathcal{F}_{N}^{K \Lambda P \Sigma}\right], \\
\tilde{\beta}^{\Phi}= & \left\{-R+4\left(\partial_{M} \Phi\right)^{2}-4 \nabla^{2} \Phi+\frac{1}{4}\left(\partial_{M} T\right)^{2}+\frac{1}{4} m^{2} T^{2}+Q^{2}\right\}, \\
\tilde{\beta}^{T}= & \left\{-\nabla^{2} T+2 T_{; M} \Phi_{;}^{M}+m^{2} T\right\}+2 e^{2 \Phi} f^{\prime}(T)\left(\mathcal{F}_{M N P \Sigma T}\right)^{2}
\end{aligned}
$$

Due to the renormalizability of the (non-critical) $\sigma$-model, there is an additional equation [18] which should supplement equation (2.5), the Curci-Paffutti relation, which relates the dilaton $\beta$-function, and hence the effective running central charge of the theory, with the rest of the $\beta$-functions:

$$
-\nabla_{M} \tilde{\beta}^{\Phi}+2 G^{N P} e^{2 \Phi} \nabla_{N}\left(e^{-2 \Phi} \tilde{\beta}_{M P}^{G}\right)+T_{; M} \tilde{\beta}^{T}=0
$$

Although this equation holds formally in the flat world sheet case, however in our framework it should also hold for the $\beta^{i}(\lambda)$ functions, i.e. the flat world sheet $\beta$-functions upon the substitution of the $\sigma$-model couplings with the Liouville dressed ones. It provides a highly non-trivial constraint [4], which should be respected by the process of identifying the 
Liouville (world-sheet) renormalization scale with the target time [5]. As emphasized by Tseytlin [17 this equation expresses, under the off-shell equivalence of the Weyl-anomaly coefficients $\tilde{\beta}^{i}$ with the field variations obtained from the target space string effective action, the invariance of the latter under general coordinate transformations. As such, the extension of this consistency condition to our Liouville string case, with the Liouville mode playing the rôle of the target time, is evident. Notice that, due to the criticality condition (3.6), the five-form $\beta$-function does not appear in (3.9).

An important comment is now in order concerning the rôle of the dilaton field in noncritical string theories. In general, it is not clear that the dilaton can be treated as one of the other couplings $g^{i}$ of the deformed $\sigma$-model. In the Appendix we specify the precise conditions under which this can indeed be made possible, thereby allowing the application of (2.5) to the dilaton coupling as well.

Upon considering the fields to be time dependent only, i.e. considering sphericallysymmetric homogeneous backgrounds, restricting ourselves to the compactification (3.4), and assuming a Robertson-Walker form of the four-dimensional metric, with scale factor $a(t)$, the generalized conformal invariance conditions (2.5), using (3.8),(3.9) imply the following equations:

$$
\begin{aligned}
& -3 \frac{\ddot{a}}{a}+\ddot{\sigma}_{1}+5 \ddot{\sigma}_{2}-2 \ddot{\Phi}+\dot{\sigma}_{1}^{2}+5 \dot{\sigma}_{2}^{2}+\frac{1}{4} \dot{T}^{2}+e^{-2 \sigma_{1}+2 \Phi} f_{5}^{2} f(T)=0 \\
& \ddot{a} a+a \dot{a}\left(2 Q+\dot{\sigma}_{1}+5 \dot{\sigma}_{2}-2 \dot{\Phi}\right)+e^{-2 \sigma_{1}+2 \Phi} f_{5}^{2} f(T) a^{2}=0 \\
& 3 \ddot{\sigma}_{1}+5 \dot{\sigma}_{1}^{2}+3 \frac{\dot{a}}{a} \dot{\sigma}_{1}+2 Q \dot{\sigma}_{1}+5 \dot{\sigma}_{1} \dot{\sigma}_{2}-2 \dot{\sigma}_{1} \dot{\Phi}+e^{-2 \sigma_{1}+2 \Phi} f_{5}^{2} f(T)=0 \\
& 3 \ddot{\sigma}_{2}+9 \dot{\sigma}_{2}^{2}+3 \frac{\dot{a}}{a} \dot{\sigma}_{2}+2 Q \dot{\sigma}_{2}+\dot{\sigma}_{1} \dot{\sigma}_{2}-2 \dot{\sigma}_{2} \dot{\Phi}-e^{-2 \sigma_{1}+2 \Phi} f_{5}^{2} f(T)=0 \\
& 2 \ddot{T}+3 \frac{\dot{a}}{a} \dot{T}+Q \dot{T}+\dot{\sigma}_{1} \dot{T}+5 \dot{\sigma}_{2} \dot{T}-2 \dot{T} \dot{\Phi}+ \\
& m^{2} T-4 e^{-2 \sigma_{1}+2 \Phi} f_{5}^{2} f^{\prime}(T)=0 \\
& \ddot{\Phi}+Q \dot{\Phi}+6 \frac{\dot{a}}{a}+6 \frac{\dot{a}^{2}}{a^{2}}+
\end{aligned}
$$




$$
\begin{aligned}
& 2\left[-\ddot{\sigma}_{1}-\frac{3 \dot{a}}{a} \sigma_{1}-5 \ddot{\sigma}_{2}-15 \frac{\dot{a}}{a} \dot{\sigma}_{2}-\dot{\sigma}_{1}^{2}-15 \dot{\sigma}_{2}^{2}-5 \dot{\sigma}_{1} \dot{\sigma}_{2}-\right. \\
& \left.2 \dot{\Phi}^{2}+2 \ddot{\Phi}+6 \frac{\dot{a}}{a} \dot{\Phi}+2 \dot{\sigma}_{1} \dot{\Phi}+10 \dot{\sigma}_{2} \dot{\Phi}\right]-\frac{1}{4} \dot{T}^{2}+\frac{1}{4} m^{2} T^{2}+Q^{2}=0, \\
& C_{5}=e^{-\sigma_{1}+5 \sigma_{2}} f(T) f_{5}, \\
& \Phi^{(3)}+Q \ddot{\Phi}+\dot{Q} \dot{\Phi}+12 \frac{\dot{a}}{a^{3}}\left(a \ddot{a}+\dot{a}^{2}+Q a \dot{a}\right)-\dot{T}(\ddot{T}+Q \dot{T})+ \\
& 4 \dot{\sigma}_{1}\left(\ddot{\sigma}_{1}+2 \dot{\sigma}_{1}^{2}+Q \dot{\sigma}_{1}\right)+20 \dot{\sigma}_{2}\left(\ddot{\sigma}_{2}+2 \dot{\sigma}_{2}^{2}+Q \dot{\sigma}_{2}\right)=0
\end{aligned}
$$

where $f^{\prime}(T)$ denotes functional differentiation with respect to the field $T$, the overdot denotes time derivative, and $\Phi^{(3)}$ denotes triple time derivative.

Note that the above equations have been derived from a ten-dimensional action. An equivalent set of equations (in fact at most linear combinations) come out from the corresponding four-dimensional action after dimensional reduction. Of course this reduction leads to the string frame. We may turn to the Einstein frame through the transformation

$$
g_{E}=e^{-2 \Phi+\sigma_{1}+5 \sigma_{2}} g
$$

In this frame the line element is

$$
d s_{E}^{2}=-e^{-2 \Phi+\sigma_{1}+5 \sigma_{2}} d t^{2}+a^{2}(t) e^{-2 \Phi+\sigma_{1}+5 \sigma_{2}}\left(d r^{2}+r^{2} d \Omega^{2}\right)
$$

Therefore to discuss cosmological evolution we have to pass to the cosmological time defined by

$$
e^{-\Phi+\frac{\sigma_{1}+5 \sigma_{2}}{2}} d t=d t_{E}
$$

Then the line element becomes

$$
d s_{E}^{2}=-d t_{E}^{2}+a^{\prime 2}\left(t_{E}\right)\left(d r^{2}+r^{2} d \Omega^{2}\right)
$$

with $a^{\prime}\left(t_{E}\right)=e^{-\Phi+\frac{\sigma_{1}+5 \sigma_{2}}{2}} a\left(t\left(t_{E}\right)\right)$.

We should also remark that we have adopted the usual Kaluza-Klein reduction without considering the extra dimensions compactified. Nevertheless the equations we are interested 
in and the results we will discuss in the following section are not modified if we had considered a compact six-dimensional space instead. In that case $e^{2 \sigma_{1}}$ and $e^{2 \sigma_{2}}$ should correspond to radii of the compact space. Note also that in the string frame we have the

exponential $e^{-2 \Phi+\sigma_{1}+5 \sigma_{2}}$ instead of $e^{-2 \Phi}$, since we allow time dependence of the volume of the compact space.

\section{Solution of the System of Differential Equations}

The problem of initial conditions of the Universe is a very complicated problem in Cosmology, and the present four-dimensional model is no exception. To bypass this problem we adopt here a different strategy. We demand that the solution approach asymptotically the Minkowski space time (in the sigma-model framework), with a linear dilaton. This space time is well-studied from the point of view of non-critical string theory [15], and provides an example of a linearly expanding, non accelerating Universe in Einstein frame日. From the point of view of a local field theory, the fact that the acceleration of the type0-string Universe is not eternal is essential for a consistent quantization, and definition of asymptotic states, and hence a scattering matrix [20, 21, 22].

We therefore assume that the universe of a type-0 non-critical string theory is described by a configuration, which, in the $\sigma$-model frame, asymptotically (i.e. for large times) approaches a Minkowski spacetime with linear dilaton and constant values of $\sigma_{1}$ and $\sigma_{2}$, and examine whether such an assumption leads to a consistent (and continuous) solution of the system of equations (3.10) for all times. For this purpose we separate the fields in their asymptotic values plus fields which tend asymptotically to zero. Substituting these

\footnotetext{
${ }^{1}$ Concerning the model of [15] there has been some discussion in the literature [19] as to whether this type of universe is really (physically) expanding or is actually static, in case one accepts the point of view that the length measurements are made by string rods. In our context there is no such ambiguity, because, as we shall see below, this type of Universe is the final stage of the Liouville-time evolution, and for us the Einstein frame, in which one recovers the linearly expanding Universe of [15], is the physical frame.
} 
fields back to the equations we let the system evolve in time backwards.

We now describe the various steps of this procedure in detail. The separation of the various fields in their asymptotic values plus fields which tend asymptotically to zero, is performed as follows:

$$
\begin{aligned}
\Phi(t) & \equiv f_{0}+f_{1} t+h(t) \\
a(t) & \equiv a_{0} e^{b(t)} \\
\sigma_{1}(t) & \equiv s_{01}+s_{1}(t) \\
\sigma_{2}(t) & \equiv s_{02}+s_{2}(t) \\
Q(t) & \equiv q_{0}+q_{1}(t) \\
T(t) & \equiv c_{0}+\mathcal{T}_{0}(t) \\
F_{5}(t) & \equiv e^{\Phi} f_{5}(t)
\end{aligned}
$$

where the constants $q_{0}$ and $f_{1}$ are related through the relation $q_{0}=-\frac{f_{1}}{2}\{1+\sqrt{17}\}$. This relation results from the requirement that the dilaton equation is satisfied. The fields $\left\{h(t), b(t), s_{1}(t), s_{2}(t), q_{1}(t), \mathcal{T}_{0}(t), f_{5}(t)\right\}$ vanish asymptotically. We want gravity to be weak asymptotically so we choose $f_{1}$ to be negative. Due to the bare tachyonic mass, $m^{0}<0$, $c_{0}$ must be chosen to vanish for the tachyon equation to be satisfied asymptotically. Note that the above asymptotic conditions in the Einstein frame cosmology correspond to a linear expanding universe [15]. With this choice the system of the equations (3.10) become quasi-linear and, in order to reduce the order of the system, we define

$$
\begin{aligned}
h(t) & \equiv h_{0}(t) \\
\dot{h}(t) & \equiv h_{1}(t) \\
b(t) & \equiv b_{0}(t) \\
\dot{b}(t) & \equiv b_{1}(t)
\end{aligned}
$$




$$
\begin{aligned}
& s_{1}(t) \equiv s_{10}(t) \\
& \dot{s}_{1}(t) \equiv s_{11}(t) \\
& s_{2}(t) \equiv s_{20}(t) \\
& \dot{s_{2}}(t) \equiv s_{21}(t) \\
& \mathcal{T}(t) \equiv \mathcal{T}_{0}(t) \\
& \dot{\mathcal{T}}(t) \equiv \mathcal{T}_{1}(t)
\end{aligned}
$$

So the system of equations $(3.10)$ take the form

$$
\dot{\vec{x}}=\mathbf{A} \vec{x}+\overrightarrow{\mathbf{F}}(\vec{x})
$$

where $\vec{x}=\left\{h_{0}(t), h_{1}(t), q_{1}(t), b_{0}(t), b_{1}(t), s_{10}(t), s_{11}(t), s_{20}(t), s_{21}(t), \mathcal{T}_{0}(t), \mathcal{T}_{1}(t), f_{5}(t)\right\}^{\perp}$, with $\perp$ denoting transpose, $\mathbf{A}$ is the constant matrix determining the linear part of the system and $\overrightarrow{\mathbf{F}}(\vec{x})$ gives the nonlinear terms.

The differential system (4.3) can be written formally as an integral system

$$
\vec{x}(t)=\vec{x}\left(t_{0}\right)+\mathbf{Y}(t) \int_{t_{0}}^{t} d s \mathbf{Y}^{-1}(s) \overrightarrow{\mathbf{F}}[\vec{x}(s)],
$$

where the matrix $\mathbf{Y}$ is a fundamental solution of the linear system satisfying the equation

$$
\dot{\mathbf{Y}}=\mathrm{AY}
$$

The full solution of this integral system can be given in an iterative form:

$$
\vec{x}_{(n+1)}(t)=\vec{x}_{(n)}(t)+\mathbf{Y}(t) \int_{t_{0}}^{t} d s \mathbf{Y}^{-1}(s) \overrightarrow{\mathbf{F}}\left[\vec{x}_{(n)}(s)\right]
$$

The starting point of the iteration procedure is the solution of the linear system with the correct asymptotic behaviour. If we insert the fields (4.1) and (4.2) into the system of equations (3.10) and keep only the linear part we get the following general solution of the system

$$
h(t)=C h_{0}+C h_{1} t+\frac{3}{4} \frac{\left(C s_{11}+5 C s_{21}\right)}{\left(f_{1}-q_{0}\right)} e^{\frac{2}{3}\left(f_{1}-q_{0}\right) t}
$$




$$
\begin{aligned}
& -\frac{3}{4} \frac{C b_{1}}{\left(f_{1}-q_{0}\right)} e^{2\left(f_{1}-q_{0}\right) t}+\frac{C_{5} C f_{5}\left(6 f_{1}^{2}+9 f_{1} q_{0}+q_{0}^{2}\right) e^{f_{0}-s_{01}-5 s_{02}+2 f_{1} t}}{8 f_{1} q_{0}\left(2 f_{1}+q_{0}\right)} \\
q_{1}(t) & =C q_{1}+\frac{3 C b_{1}\left(2 f_{1}-q_{0}\right)}{2 f_{1}} e^{2\left(f_{1}-q_{0}\right) t} \\
& -\frac{\left(C s_{11}+5 C s_{21}\right)\left(2 f_{1}+q_{0}\right)}{6 f_{1}} e^{\frac{2}{3}\left(f_{1}-q_{0}\right) t} \\
& -\frac{C_{5} C f_{5}\left(6 f_{1}^{2}+9 f_{1} q_{0}+q_{0}^{2}\right)}{8 f_{1}^{2} q_{0}} e^{f_{0}-s_{01}-5 s_{02}+2 f_{1} t} \\
b(t) & =C b_{0}+\frac{C b_{1}}{2\left(f_{1}-q_{0}\right)} e^{2\left(f_{1}-q_{0}\right) t}-\frac{C_{5} C f_{5}}{8 f_{1} q_{0}} e^{f_{0}-s_{01}-5 s_{02}+2 f_{1} t} \\
s_{1}(t) & =C s_{10}+\frac{3 C s_{11}}{2\left(f_{1}-q_{0}\right)} e^{\frac{2}{3}\left(f_{1}-q_{0}\right) t}-\frac{C_{5} C f_{5}}{8 f_{1}\left(2 f_{1}+q_{0}\right)} e^{f_{0}-s_{01}-5 s_{02}+2 f_{1} t} \\
s_{2}(t) & =C_{20}+\frac{3 C s_{21}}{2\left(f_{1}-q_{0}\right)} e^{\frac{2}{3}\left(f_{1}-q_{0}\right) t}+\frac{C_{5} C f_{5}}{8 f_{1}\left(2 f_{1}+q_{0}\right)} e^{f_{0}-s_{01}-5 s_{02}+2 f_{1} t} \\
F_{5}(t) & =C f_{5} e^{f_{1} t} \\
\mathcal{T}_{0}(t) & =C_{1} e^{A t}+C_{2} e^{B t}+\frac{C f_{5}}{8 f_{1}^{2}+2 m^{2}+4 f_{1} q_{0}} e^{f_{0}-s_{01}-5 s_{02}+2 f_{1} t}
\end{aligned}
$$

where

$$
\begin{aligned}
& A \equiv \frac{1}{4}\left[\left(2 f_{1}-q_{0}\right) \sqrt{\left(2 f_{1}-q_{0}\right)^{2}-8 m^{2}}\right]<0 \\
& B \equiv \frac{1}{4}\left[\left(2 f_{1}+q_{0}\right) \sqrt{\left(2 f_{1}-q_{0}\right)^{2}-8 m^{2}}\right]>0
\end{aligned}
$$

and $\left\{C h_{0}, C h_{1}, C q_{1}, C b_{0}, C b_{1}, C s_{10}, C s_{11}, C s_{20}, C s_{22}, C_{1}, C_{2}, C f_{5}\right\}$ are constants of integration. To have the right asymptotic behaviour we choose $\left\{C h_{0}, C h_{1}, C b_{0}, C q_{1}, C s_{10}, C s_{20}\right\}$ to be zero. The constant $C_{2}$ corresponds to a positive eigenvalue of the matrix $\mathbf{A}$, so we set it also zero. Since the constant matrix A has negative eigenvalues, well known theorems from qualitative theory of quasi-linear systems, guarantee the asymptotic stability of the iterative solution [23]

Now at the first step in the iteration the solution is given by:

$$
\vec{x}(t)=\vec{x}_{(0)}(t)+\mathbf{Y}(t) \int_{t_{0}}^{t} d s \mathbf{Y}^{-1}(s) \overrightarrow{\mathbf{F}}[\mathbf{Y}(s) \vec{C}]
$$


where $\vec{x}_{(0)}(t)$ is given by,

$$
\vec{x}_{(0)}(t)=\mathbf{Y}(t) \vec{C}
$$

and $\vec{C}$ is a constant vector $\vec{C}=\left\{C h_{0}, C h_{1}, C q_{1}, C b_{0}, C b_{1}, C s_{10}, C s_{11}, C s_{20}, C s_{22}, C_{1}, C_{2}, C f_{5}\right\}$ with $C h_{0}=C h_{1}=C q_{1}=C b_{0}=C s_{10}=C s_{20}=C_{2}=0$ for reasons we explained above.

The first step of the iteration can be integrated analytically and has the right asymptotic behaviour as expected. Nevertheless due to the complexity of the expressions and the fact that they do not give us the full solution of the problem, we proceed as follows. We take from this step the value of the fields at late times and we use them as initial conditions for the numerical solution of the system.

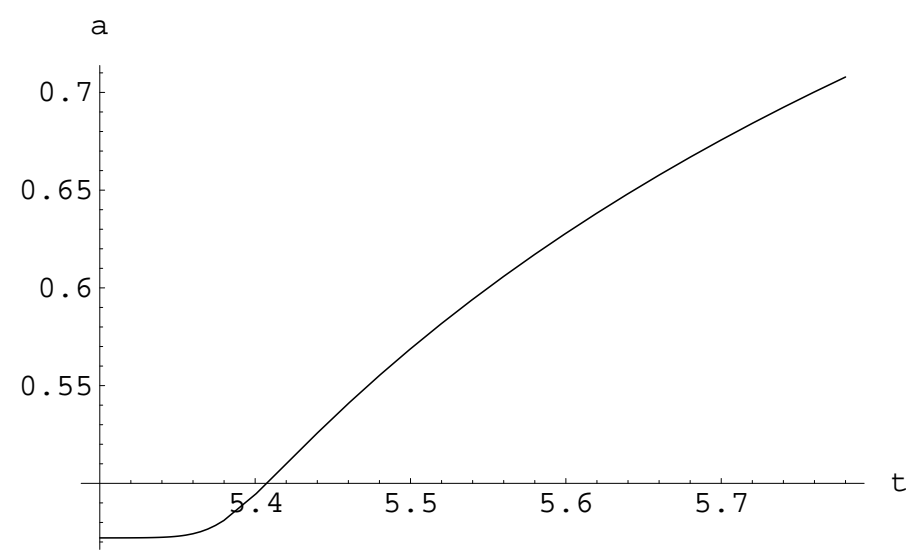

Figure 1: The evolution of the scale factor in time.

We can now discuss our results. As we can see from Fig.1-Fig.3, the cosmological evolution of our universe passes through the following phases. At very early times, the universe starts from the initial singularity. Then enters a phase where the physical dimensions are formed. At this stage $\sigma_{1}, \sigma_{2}$ and $a$ are comparable in magnitude. The phase of inflation follows, during which the scale factor grows exponentially (for a short time though), while the internal space contracts with very negative values of $\ddot{\sigma}_{1}$ and $\ddot{\sigma_{2}}$. Finally, the universe enters a phase, where its expansion slows down until it reaches the asymptotically flat space (in the $\sigma$-model frame). The internal space continues to contract but with very slow rate (with $\ddot{\sigma_{1}}$ and $\ddot{\sigma_{2}}$ positive) until it reaches a constant value. As we can see from Fig.2 and 
Fig.3, $\sigma_{1}$ and $\sigma_{2}$ scale differently. The field $\sigma_{2}$ very soon freezes to a value much lower than the value of $\sigma_{1}$, indicating the the fifth dimension can be much larger than the other five dimensions.

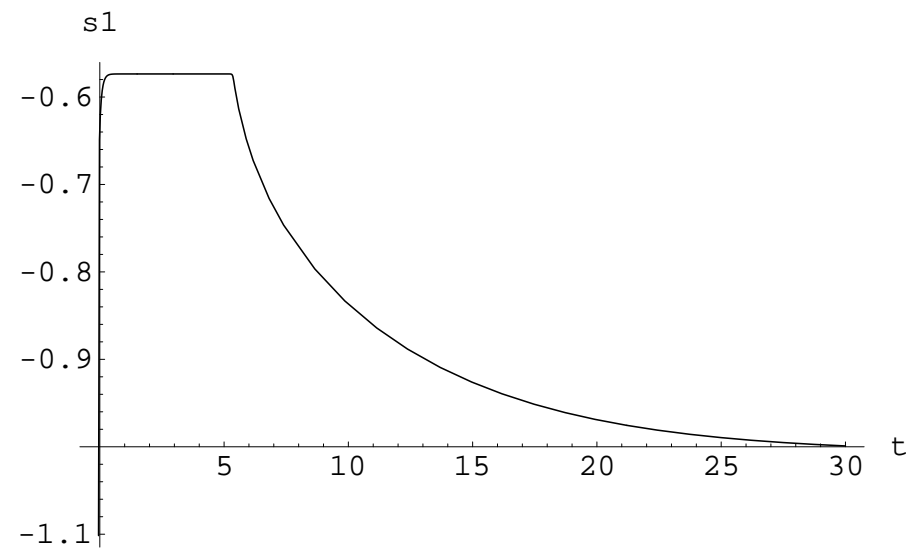

Figure 2: The behaviour of the field representing the evolution of the fifth dimension.

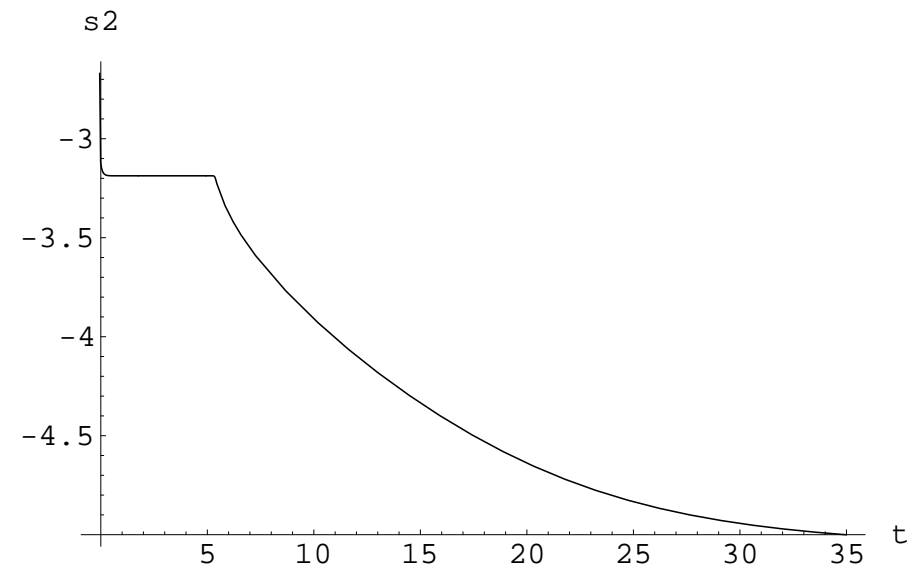

Figure 3: The behaviour of the field $\sigma_{2}$ expressing the evolution of the five internal dimensions. The difference of the scales in the decomposition of the extra dimensions is clearly seen.

For the dilaton field (Fig.4), we observe that at the singularity the dilaton field is infinite, indicating that the gravity is very strong. Then at the second phase of evolution, the strength of gravity is weakened because the dilaton field drops linearly. This weakening of the gravitational interactions continues during inflation, and finally at the exit, the 
dilaton field continues to drop linearly. The Tachyon field (Fig.5) falls continuously during the evolution until it becomes zero. The RR-field (Fig. 6), on the other hand, grows from zero value at the initial singularity until it reaches a constant value at the exit of inflation. Moreover, the behaviour of the central-charge deficit $Q^{2}$ is depicted in figure 7 . The latter is initially decreasing, until it becomes zero, and then oscillates before reaching an asymptotic constant positive value $Q^{2} \rightarrow$ const $\neq 0$, as $t \rightarrow \infty$. A similar behaviour is observed in the two dimensional case [4]. Notice that the oscillations in the central charge deficit are compatible with the fact that the Liouville mode is not a unitary $\sigma$-model field, as a result of its negative signature. Hence, Zamolodchikov's $c$-theorem does not strictly apply, although overall there is a reduction in the central charge, since the theory starts with a very large value of $Q^{2}$ (formally infinite near the initial singularity), which eventually asymptotes to a finite positive value.

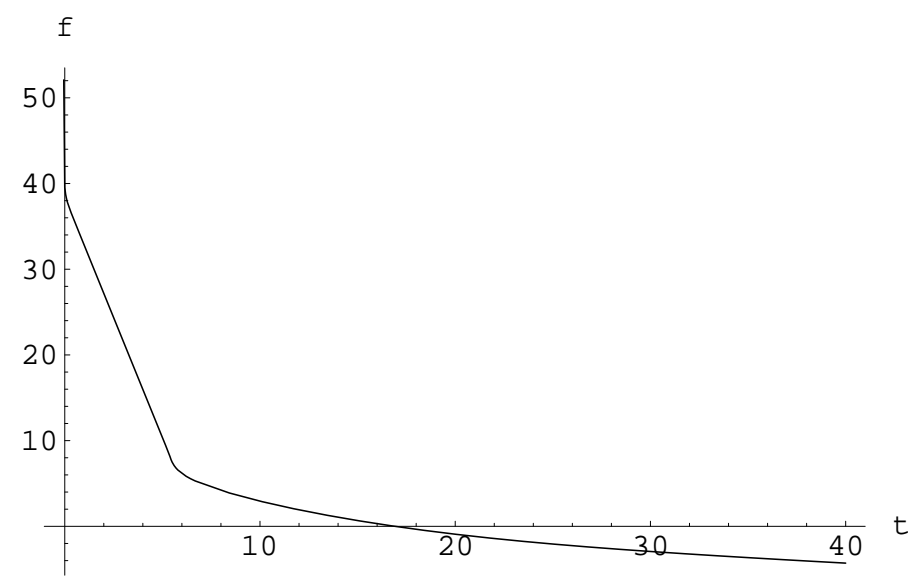

Figure 4: The behaviour of the dilaton field.

We can analytically support the numerical solution we found looking more carefully at the equations satisfied by the fields. Assuming that asymptotically the fields $\sigma_{1}$ and $\sigma_{2}$ are constant, using the second and seventh equation from the system of equations (3.10), the field $b$ takes the form of a Riccati equation, which is the usual case in cosmological models 


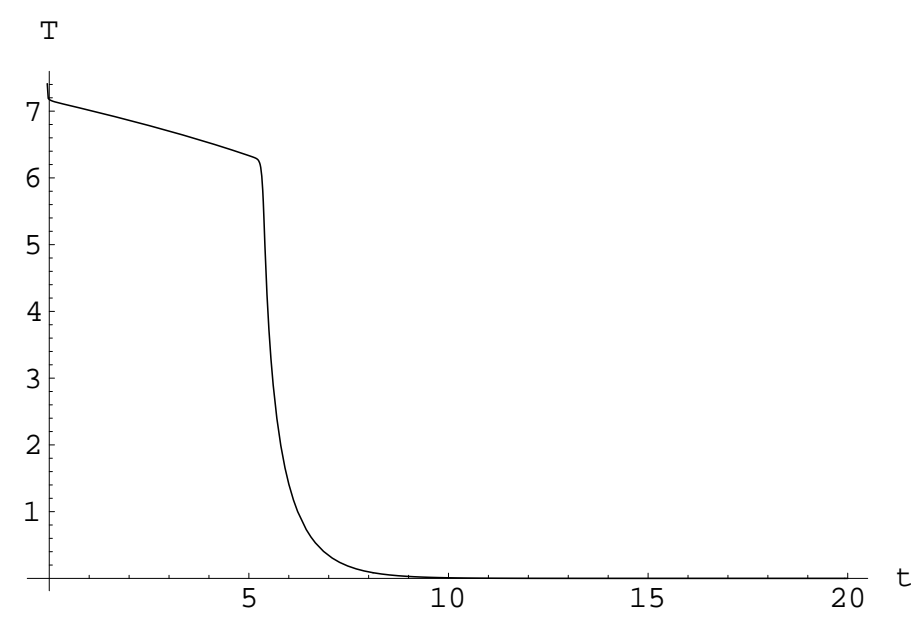

Figure 5: The behaviour of the tachyon field.



Figure 6: The evolution of the field $f_{5}$ denoting the behaviour of the $\mathrm{R}$ - $\mathrm{R}$ flux.

based on type-0 string theory [2],

$$
\ddot{b}+\dot{b}\left(2 Q-2 \dot{\Phi}+\dot{\sigma}_{1}+5 \dot{\sigma_{2}}\right)+\dot{b}^{2}=-\frac{C_{5}}{2} e^{-\sigma_{1}+5 \sigma_{2}+\Phi} F_{5}
$$

For times nearly after the initial singularity and during inflation, equation (4.11) is approximated by

$$
\ddot{b}+2 \dot{b}(Q(t)-\dot{\Phi})+\dot{b}^{2}=0
$$

This equation has the solution

$$
b=c_{1}+t+\ln \left(1-e^{-c_{2} t}\right)
$$




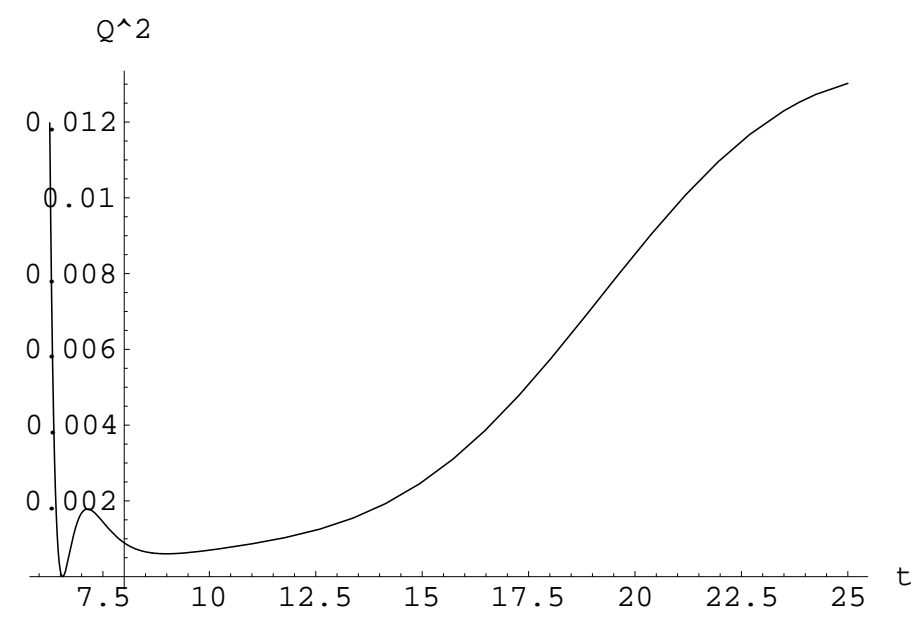

Figure 7: The behaviour of the central charge deficit $Q^{2}$. This quantity, after some oscillatory behaviour near its zero value, asymptotes for large times to a constant value (not depicted clearly in the figure due to numerical limitations).

where $c_{1}, c_{2}$ are constants. This solution shows that the scale factor has an inflationary phase and for later times the logarithmic term takes over and slows down the expansion of the universe.

For large times when the internal dimensions have been stabilized in constant values, the equation (4.11) becomes

$$
\ddot{b}+2 \dot{b}\left(q_{0}-f_{1}\right)+\dot{b}^{2}=c e^{2 f_{1} t}
$$

The exact solution of this equation respecting the required asymptotic behaviour is

$$
b(t)=\ln \left[{ }_{0} F_{1}\left(\frac{q_{0}}{f_{1}}, \frac{c}{4 f_{1}^{2}} e^{2 f_{1} t}\right)+c_{3} e^{2\left(f_{1}-q_{0}\right) t}{ }_{0} F_{1}\left(2-\frac{q_{0}}{f_{1}}, \frac{c}{4 f_{1}^{2}} e^{2 f_{1} t}\right)\right]
$$

where $c_{3}$ is a constant, and ${ }_{0} F_{1}$ is the Confluent Hypergeometric Function. If we expand this solution in series, we get the asymptotic behaviour of $b(t)$ which characterizes the inflationary exit period. The quantity $Q(t)-\dot{\Phi}$ is crucial for the existence of an inflationary phase and then exit from it. We note that in two target dimensions, it is the same quantity that controls the exit from inflation [4]. 
We now make some brief remarks on the physical significance of the asymptotic behaviour of our solution (for large times $t \rightarrow \infty$ ). A detailed discussion will appear in a forthcoming publication [24]. By inspecting figure 1, or analytically from (4.15), we observe that the acceleration of the universe vanishes asymptotically in time $(t \rightarrow \infty)$ and one recovers a non-accelerating Minkowski universe with a linear dilaton in the $\sigma$-model (string) frame [15]: $\ddot{a} a=\ddot{b}+(\dot{b})^{2} \rightarrow 0$, for $t \rightarrow \infty$, with $a(t) \rightarrow a_{o}$.

Such configurations imply the absence of a cosmological horizons, given that the relevant quantity diverges, $\delta \propto \int_{t_{0}}^{\infty} d t / a(t) \rightarrow \infty$. This is a welcome fact from the point of view of low-energy field theory, in the sense that in such not-eternally accelerating Universes one is able to define asymptotic states and thus a proper $S$ matrix [20]. Out of interest we notice that asymptotic situations of this kind appear to be generic in cosmologies with fluctuating D-brane defects $\llbracket 7$.

Another issue that arises in this scenario is the asymptotic value of the vacuum energy. In the Einstein frame (3.13), the latter is given by

$$
\Lambda=e^{2 \Phi-\sigma_{1}-5 \sigma_{2}} Q^{2} \rightarrow e^{-|c o n s t| t-\sigma_{1}-5 \sigma_{2}} Q^{2} \rightarrow 0, \quad t \rightarrow \infty
$$

where we took into account that asymptotically in time the fields $\sigma_{1}, \sigma_{2}$ become constants, the dilaton configuration is linear and negative (see figure 4), while the central-charge deficit $Q^{2} \rightarrow$ const $>0$. In our solution, therefore, we observe that the four-dimensional vacuum energy is positive (de Sitter type) and tends to zero asymptotically. When we express the result (4.16) in terms of the Robertson-Walker time $t_{E}(3.14)$ we observe that the four-dimensional vacuum energy relaxes to zero as:

$$
\Lambda=(\text { const }) \frac{1}{t_{E}^{2}}
$$

\footnotetext{
${ }^{2}$ Notice that a divergent result for $\delta$ is, of course, also obtained in the Einstein frame (3.13), (3.14), when we express the integrand in terms of the Robertson-Walker time $t_{E}$. In that case the scale factor is expanding linearly $a^{\prime}\left(t_{E}\right) \propto t_{E}$, while the dilaton varies logarithmically with the time $t_{E}$.
} 
which is compatible with recent experimental evidence, and is obtained in many quintessence models. In our approach the quintessence field is, in a sense, the dilaton [13, 21]. We shall present a more detailed account of these features in a forthcoming publication [24].

We next remark that in the context of a critical string theory, with $Q=0$, the dilaton equation in (3.10) implies that the dilaton field $\Phi$ becomes asymptotically constant and, hence, equation (4.14) becomes

$$
\ddot{b}+\dot{b}^{2}=c
$$

This has as solution

$$
b(t)=c_{1}+\ln \left[\cosh \left\{\sqrt{c} t-\sqrt{c} c_{2}\right\}\right]
$$

where $c_{1}$ and $c_{2}$ are constants. Then the scale factor has an exponential growth but it will expand for ever. Such a case is inconsistent from the point of view of defining asymptotic states and an $S$-matrix. Because the case $Q=0$, is supposed to correspond to critical string theory, which must be a theory of an $S$-matrix, we may conclude that such a situation will not arise as a consistent string theory solution.

All the analysis we have obtained so far pertains to the string ( $\sigma$-model) frame. As already mentioned, one gets similar results in the Einstein frame, using (3.13) and (3.14).

Another consistency check of the approach is provided by the F-field of the RR-flux. Because of equation (3.6), the field $F_{5}(t)$ has the exact solution

$$
F_{5}(t)=e^{\phi} f_{5}=\frac{C_{5} e^{f_{0}+s_{01}-5 s_{02}}}{\left(1+g(t)+\frac{g(t)^{2}}{2}\right)} e^{f_{1} t+h(t)}
$$

Stability of the internal space and consistency of our scheme requires the constant $f_{0}$ to have a large negative value. This means that gravity is asymptotically very weak, as expected.

\section{Discussion}

In the present article we have presented a cosmological model based on a type-0 string theory. The type- 0 string theory is rich in its content. In addition to the graviton and 
dilaton fields, it includes also a tachyon field which couples to an RR five-form field. To avoid tachyon instabilities the tachyon field has to take on values at the minimum of its potential. Considering the ten-dimensional action of the type- 0 field theory one can get at the conformal point the $\beta$-functions of the theory, and from them, by reduction to four-dimensions, and assuming that all fields are time dependent, one obtains an effective four-dimensional theory. It is found that this theory, in the context of a Robertson-Walker background, has an inflationary phase but there is no smooth exit from this phase.

We believe that the central issue of inflationary cosmology is not how one can get an inflationary phase, but how one can exit from it. In view of the recent discussion 20 of the exit from inflation in string theory, and in general on the consistent quantization of de-Sitter Universes [22], we think that it is useful to propose new mechanisms to exit from inflation. In our previous work [4], we had proposed a mechanism based on non-critical strings, to exit from inflation. We had considered a two dimensional model with a tachyon field. The presence of the tachyon field was crucial for an inflationary phase to go smoothly to a Robertson-Walker phase. Notably, in two-dimensions the tachyon field is an excitation of the 'massless' matter multiplet, and is not associated with an instability of the vacuum.

In the present work we have applied this mechanism to a realistic four-dimensional model of type 0 string theory, where again the tachyon is stabilized by a RR flux field. We modified the $\beta$-functions of the ten-dimensional theory, assuming that the string theory is non-critical, which in such theories may be caused by fluctuations of the 3-brane worlds. This hypothesis introduces new terms in the $\beta$-functions of the theory. Physically these terms express the fact that our non-critical string theory is performing small oscillations around the conformal point.

The modified $\beta$-functions supplemented with the Curci-Paffuti equation implied by world-sheet renormalizability, have been reduced to four-dimensions. Assuming a homogeneous and spherically symmetric background, we have solved numerically the resulting 
equations. Our solution demonstrates that the scale factor of the Universe, after the initial singularity, enters a short inflationary phase and then, in a smooth way, goes onto a flat Minkowski spacetime with a linear dilaton for $t \rightarrow \infty$. The fields $\sigma_{1}$ and $\sigma_{2}$ which parametrise the internal space have an interesting behaviour. The field $\sigma_{1}$, which sets the scale of the fifth dimension, during inflation contracts until it reaches a constant value. After inflation, it maintains this value, until the universe evolves to an asymptotically flat spacetime (in the string frame, or a linearly expanding, non-accelerating Universe in the Einstein frame). The field $\sigma_{2}$ which parametrises the conformally flat five-dimensional space freezes to a constant value which is much smaller than that of the fifth dimension. Thus we see that, in our model, a cosmological evolution may lead to different scales for the extra dimensions.

It is important to notice that the contraction of the extra dimensions is due to the fact that gravity is very weak asymptotically in our model. It is also worthy of stressing that the possibility of having one large extra dimension is achieved upon the choice of the RR flux along that direction. Hence, such a possibility arises in our model from string non-perturbative effects. A phenomenologically important feature of the model is that the vacuum energy, determined by the central-charge deficit, relaxes to zero asymptotically in a way which is reminiscent of quintessence models, with the rôle of the quintessence field played by the dilaton.

The type-0 string Universe does not accelerate eternally, thus avoiding the problem associated with the presence of cosmological horizons, as far as the definition of proper asymptotic states, and hence an S-matrix, is concerned. A detailed study of such issues, as well as of re-heating and other physical features of inflationary models, will be the subject of forthcoming work.

We close our discussion with an important comment concerning the initial singularity of our solution. The singularity is a general feature of the equations of the form (3.10). 
Removing the singularity is probably a matter of a full quantum description of the theory, which at present is not available. We note at this stage, however, the possibility of deriving smooth cosmological solutions in string theory, without initial singularities, by including in the action higher curvature terms (e.g. quadratic of Gauss-Bonnet type 25]), which are part of the quantum corrections, in the sense of being generated by including string-loop corrections in the effective action. We plan to return to a systematic study of the effects of such higher-order terms, within our framework, in due course.

\section{Acknowledgements}

The work of E.P and I.P is partially supported by the NTUA program Archimedes. G.A.D and B.C.G. would like to acknowledge partial financial support from the Athens University special account for research.

\section{Appendix}

\section{Dilaton Equations in Liouville Strings}

In this Appendix we explain in some detail why the dilaton field obeys an equation of the form (2.5), in a similar manner with the rest of the background fields/couplings of the deformed $\sigma$-model. At a $\sigma$-model level, the dilaton field couples to the world-sheet curvature in the same way as the Liouville mode, which implies that in general the dilaton of the Liouville-dressed theory will receive contributions from the Liouville mode $\rho$. The Liouville mode $\rho$ is viewed as an extra target space dimension, which has Minkowskian signature in supercritical theories [15]. In our approach, we first treat this extra time dimension as a second time, which, however, is eventually going to be identified with the existing time-coordinate $t$ of the non-critical string.

In this sense, the restoration of conformal invariance implied by the Liouville dressing [6] 
means that in $D+1$ dimensions, with $D$ the original dimensionality of the non-critical theory, one would have a conformal string. In terms of the $\tilde{\beta}^{\Phi}$ function we would have: $\tilde{\beta}^{\Phi(D+1)}=C_{\mathrm{tot}}^{(D+1)}-10=0$, where $C_{\mathrm{tot}}^{(D+1)}$ is the total central charge of the Liouville-dressed theory, and the superscript $D+1$ denotes $(D+1)$-dimensional quantities. To order $\mathcal{O}\left(\alpha^{\prime}\right)$ in a Regge slope $\alpha^{\prime}$ expansion, one has for a $d$-dimensional target space:

$$
C_{\mathrm{tot}}^{(d)}=d-\frac{3}{2} \alpha^{\prime}\left[R-4(\nabla \Phi)^{2}+4 \nabla^{2} \Phi+\frac{1}{4}(\nabla T)^{2}+\frac{1}{4} m^{2} T^{2}\right]
$$

In general, for non-critical strings with central-charge deficit $Q^{2}$, one should replace $d$ by $3\left(Q^{2}+d^{*}\right)$, where $d^{*}$ is the critical value of the central charge for the conformal (fixed-point) theory $\left(d^{*}=10\right.$ for superstrings we are dealing with here). For convenience we set from now on $\alpha^{\prime}=2$, which is the normalization used in (3.8) and throughout this work.

One now recalls the definition $\tilde{\beta}^{\Phi(D+1)}=\beta^{\Phi(D+1)}-G^{\mu \nu} \beta_{\mu \nu}^{G(D+1)}$, where $\mu, \nu$ span the $D+1$-dimensional spacetime, including the Liouville dimension $\rho$. In Liouville theory, the metric $G_{\rho \rho}=-1, G_{\rho M}=0$, and hence $\beta_{\rho \rho}^{G}=0=\beta_{\rho M}^{G}$, which implies that the Liouville renormalization does not introduce any extra contributions to the trace of the graviton $\beta$ function, and therefore to the space-time curvature terms in the expression for $\tilde{\beta}^{\Phi}$ (3.8). This leaves one with the following condition, expressing restoration of conformal invariance:

$$
4 G^{\rho \rho}\left[\left(\partial_{\rho} \Phi\right)\left(\partial_{\rho} \Phi\right)-\partial_{\rho} \partial_{\rho} \Phi-\frac{1}{2} G^{M N}\left(\partial_{\rho} G_{M N}\right)\left(\partial_{\rho} \Phi\right)+\frac{1}{16}\left(\partial_{\rho} T\right)\left(\partial_{\rho} T\right)\right]+\tilde{\beta}^{\Phi(D)}=0
$$

where $\tilde{\beta}^{\Phi(D)}$ refers to the $D$-dimensional parts of the dilaton Weyl anomaly coefficient (3.8), which notably include the dilaton field.

As we shall discuss below, the terms $\frac{1}{2} G^{M N} \partial_{\rho} G_{M N} \partial_{\rho} \Phi$ may be neglected. There are two ways in which this can be justified. First, in our approach [5] we treat the Liouville mode $\rho$ as a world-sheet renormalization-group scale. Hence terms like $\partial_{\rho} G_{M N}=\beta_{M N}^{G}$ and $\partial_{\rho} \Phi=\beta^{\Phi}$ coincide with the world-sheet graviton and dilaton $\beta$-functions. Thus, such terms are of higher order, as compared with the rest of the terms in (5.2), if one works in the neighbourhood of a fixed point, which we assume here. Alternatively, such quadratic in 
the $\beta$-functions terms can be eliminated by an appropriate renormalization-group scheme choice [26], as we shall discuss later.

We now assume that the Liouville mode $\rho$ contributions to $\Phi(\rho, t, \ldots)$ can be represented by

$$
\Phi(\rho, t, \ldots)=-\gamma Q(\rho) \rho+\varphi(\rho, t, \ldots)
$$

where the ... denote possible dependence on spatial target coordinates, $\gamma$ is a numerical constant, to be determined below, and the term proportional to $Q \rho$ is motivated by the Liouville dynamics [6, 5]. Under this splitting, and taking into account that $Q$ does not depend on any other coordinate except the local renormalization-group scale $\rho$ [5], one may write (5.2) for the supercritical string case $G^{\rho \rho}=-1$ :

$$
4 \ddot{\varphi}-4 \gamma^{2} Q^{2}+8 \gamma Q \dot{\varphi}=-\tilde{\beta}^{\Phi}+\mathcal{O}\left[\left(\partial_{\rho} \Phi\right)^{2},\left(\partial_{\rho} T\right)^{2}, \partial_{\rho} Q,\left(\partial_{\rho} G\right)\left(\partial_{\rho} \Phi\right)\right]
$$

where $\partial_{\rho} Q \propto\left(\beta^{i}\right)^{2}$ by virtue of the Zamolodchikov $c$-theorem [11], extended to the local scale $\rho$ [5].

Then, terms of the form $\mathcal{O}\left[\left(\partial_{\rho} \varphi\right)^{2},\left(\partial_{\rho} T\right)^{2}, \partial_{\rho} Q,\left(\partial_{\rho} G\right)\left(\partial_{\rho} \Phi\right)\right]$ can be removed by field redefinitions (appropriate renormalization-group scheme choice). This is possible due to the gradient flow property (2.6) of the $\beta$-functions [26], which can be shown [5] to characterize the Liouville problem upon considering [5] the Liouville mode as a local scale on the world-sheet, appropriate for curved-(two-dimensional)-space renormalization [12]. Indeed, consider an infinitesimal field-redefinition $\delta g^{i}$ (or equivalently $\delta \lambda^{i}$ for the Liouville-dressed couplings), where now the set $g^{i}$ includes the dilaton field. This expresses a particular choice of (a class) of renormalization group schemes. Under this redefinition, the central charge deficit $Q^{2}$, which is assumed to satisfy the gradient flow property $\partial_{i}\left(Q^{2}\right)=\mathcal{G}_{i j} \beta^{j}$, changes as

$$
\delta\left(Q^{2}\right)=\delta g^{i} \partial_{i}\left(Q^{2}\right)=\delta g^{i} \mathcal{G}_{i j} \beta^{j}
$$

It is then clear that by choosing appropriately $\delta g^{i} \propto \beta^{i}$, one may absorb terms of the form $\mathcal{O}\left[\left(\partial_{\rho} \varphi\right)^{2},\left(\partial_{\rho} T\right)^{2}, \partial_{\rho} Q,\left(\partial_{\rho} G\right)\left(\partial_{\rho} \Phi\right)\right]$ on the right-hand-side of (5.4) 26]. Moreover, as 
already mentioned, such terms may also be neglected due the fact that, in the models of 'recoil' fluctuations of D3-brane worlds we are dealing with here [7, 8], the induced noncriticality is marginal, thereby forcing us to work in the neighbourhood of a world-sheet renormalization-group fixed point. Hence, terms quadratic in the $\beta$-functions, and thus in $\partial_{\rho} \lambda^{i}$, are subdominant compared to terms linear in such quantities.

With the above in mind, we now observe that, upon identifying $\rho=2 t$, where $t$ is the target time, fixing $\gamma=1 / 4$, and re-expressing the terms involving $\varphi$ in terms of $\Phi$, we may rewrite (5.4) in the form (2.5), up to terms (denoted by ...), which may either be removed by a renormalization-scheme choice or are negligible in magnitude for an expansion in the neighbourhood of a fixed point we are assuming throughout:

$$
0=\ddot{\varphi}+Q \dot{\varphi}+\tilde{\beta}^{\Phi}-\frac{1}{4} Q^{2}=\ddot{\Phi}+Q \dot{\Phi}+\tilde{\beta}^{\Phi}+\ldots=0
$$

The overdot denotes differentiation with respect to time $t$. Notice that the identification of the local world-sheet renormalization-group scale $\rho$ with the target time may be understood as a renormalization-group scheme choice [5].

Thus, in this way, the dilaton equation (5.6) is now expressed as an equation of the form (2.5). Therefore, by an appropriate scheme choice, one was able use the equations (2.5) for all $\sigma$-model couplings, including the dilaton, in a way consistent with both the restoration of conformal invariance by the Liouville mode, and the identification of the latter with the target time.

\section{References}

[1] I. Klebanov and A.A. Tseytlin, Nucl. Phys. B546, 155 (1999); Nucl. Phys. B547, 143 (1999).

[2] E. Papantonopoulos and I. Pappa, Mod. Phys. Lett. A15 , 2145 (2000), hepth/0001183; Phys. Rev. D63, 103506, (2001), hep-th/0103101; and gr-qc/0103101. 
[3] G. A. Diamandis, B. C. Georgalas and E. Papantonopoulos, Mod. Phys. Lett. A10, $1277(1995)$

G. A. Diamandis, J. Ellis, B. C. Georgalas, N. E. Mavromatos, D. V. Nanopoulos and E. Papantonopoulos, Int. J. Mod. Phys. A13, 4265 (1998) gr-qc/9712051.

[4] G. A. Diamandis, B. C. Georgalas, N. E. Mavromatos and E. Papantonopoulos, Phys. Lett. B461, 57 (1999) hep-th/9903045.

[5] J. Ellis, N. E. Mavromatos and D. V. Nanopoulos, Phys. Lett. B293, 37, (1992);

Erice Subnuclear Series (World Sci., Singapore) 31 1, (1993); hep-th/9304133; For a concise review see: J. Chaos, Solitons and Fractals 10, 345 (eds. C. Castro amd M.S. El Naschie, Elsevier Science, Pergamon 1999) hep-th/9805120.

[6] F. David, Mod. Phys. Lett. A3 1651 (1988);

J. Distler and H. Kawai, Nucl. Phys. B321 509 (1989);

J. Distler, Z. Hlousek and H. Kawai, Int. J. Mod. Phys. A 5, 391 (1990);

see also N.E. Mavromatos and J.L. Miramontes, Mod. Phys. lett. A4 1847 (1989).

[7] I. I. Kogan, N. E. Mavromatos and J. F. Wheater, Phys. Lett. B 387, 483 (1996) hep-th/9606102; J. R. Ellis, N. E. Mavromatos and D. V. Nanopoulos, Int. J. Mod. Phys. A 12, 2639 (1997) hep-th/9605046; N. E. Mavromatos and R. J. Szabo, Phys. Rev. D 59, 104018 (1999) hep-th/9808124; E. Gravanis and N. E. Mavromatos, hepth/0106146.

[8] N. E. Mavromatos and R. J. Szabo, hep-th/0106259;

[9] A. Campbell-Smith and N. E. Mavromatos, Phys. Lett. B 488, 199 (2000) hepth/0003262.

[10] I.R. Klebanov, I.I. Kogan and A.M. Polyakov, Phys. Rev. Lett. 71, 3243, (1993);

C. Schmidhuber, Nucl. Phys. B435, 156, (1995). 
[11] A.B. Zamolodchikov, JETP Lett. 43 (1986) 730; Sov. J. Nucl. Phys. 46 (1987) 1090.

[12] H. Osborn, Phys.Lett. B222, 97, (1989); Annals Phys. 200, 1, (1990).

[13] J. Ellis, N.E. Mavromatos and D.V. Nanopoulos, Mod. Phys. Lett. A10 (1995) 1685.

[14] C. Schmidhuber and A.A. Tseytlin, Nucl. Phys. B426, 187, (1994).

[15] I. Antoniadis, C. Bachas, John Ellis and D.V. Nanopoulos, Phys. Lett. B211 383 (1988); Nucl. Phys. B328 117 (1989).

[16] J. Ellis, P. Kanti, N.E. Mavromatos, D.V. Nanopoulos and E. Winstanley, Mod. Phys. Lett. A13, 303, (1998), and references therein;

[17] G. Shore, Nucl. Phys. B286, 349, (1987);

A. A. Tseytlin, Nucl. Phys. B294, 383, (1987); Int. J. Mod. Phys. A5, 1833, (1990).

[18] G. Curci and G. Paffuti, Nucl. Phys. B286, 399, (1987).

[19] N. Sanchez and G. Veneziano, Nucl. Phys. B 333, 253 (1990).

[20] S. Hellerman, N. Kaloper and L. Susskind, hep-th/0101180; W. Fischler, A. KashaniPoor, R. McNees, S. Paban, [hep-th/0104181]; A.P. Billyard, A.A. Coley and J.E. Lidsey, J. Math. Phys. 41 (2000) 6277.

[21] J. R. Ellis, N. E. Mavromatos and D. V. Nanopoulos, hep-th/0105206.

[22] E. Witten, hep-th/0106109

[23] S. Nemytskii and B. Stepanov, Qualitative Theory of Differential Equations (Princeton University Press, 1960).

[24] G. Diamandis, V. Georgalas, N.E. Mavromatos, E. Papantonopoulos and I. Pappa, in preparation. 
[25] See, for instance: I. Antoniadis, J. Rizos and K. Tamvakis, Nucl. Phys. B 415, 497 (1994) hep-th/9305025.

[26] N. E. Mavromatos, Mod. Phys. Lett. A 3, 1079 (1988). 